\title{
Actualización en fascitis necrotizante
}

\section{Update on necrotizing fasciitis}

Pág. 186,194

Recibido: 08-06-2020

Aceptado: 13-07-2020

Dr. Daniel Morún Calvo ${ }^{1}$

Dra. Marcela Kriebel Haehner ${ }^{2}$

Dr. Andrey González Chavarría ${ }^{3}$

Dra. Adriana Zúñiga Villegas ${ }^{4}$

1,2,3,4. Médico General, Trabajador Independiente. San José,Costa Rica.

\section{RESUMEN}

La fascitis necrotizante es una enfermedad infecciosa grave, poco frecuente y de difícil diagnóstico de los tejidos blandos. Generalmente se caracteriza por ser polimicrobiana y su manejo es difícil por la necrosis que genera. La mortalidad de esta patología es alta y la mejor manera de mejorar el pronóstico es conocer de la misma, con diagnóstico y tratamiento precoz.

\section{PALABRAS CLAVE}

fascitis necrotizante, infección de tejidos blandos, fascia

\section{ABSTRACT}

Necrotizing fasciitis is a rare and serious infectious disease that is difficult to diagnose localized in soft tissues. It is generally characterized by being polymicrobial and its management is difficult due to the necrosis it generates. The mortality of this pathology is high and the best way to improve the prognosis is knowledge about disease and the early diagnosis and treatment.

\section{KEY WORDS}

fasciitis, necrotizing, soft tissue infection, fascia

\section{INTRODUCCIÓN}

La fascitis necrotizante (FN) es una infección poco frecuente y rápidamente progresiva en los tejidos blandos, altamente destructiva que provoca necrosis de la piel, tejido subcutáneo y fascia. Debido a estas características, el diagnóstico temprano y el tratamiento oportuno son necesarios para lograr reducir la morbilidad y mortalidad que genera (1). 
En esta entidad, la presencia de necrosis de la fascia es el factor determinante, ya que se encuentra ausente en otras infecciones de tejidos blandos como la celulitis necrotizante y en la mionecrosis (2). Esta infección se diagnostica con mayor frecuencia entre la cuarta y sétima década, con un predominio en hombres. Su incidencia es de 0,4 por cada 100,000 habitantes. La mortalidad global oscila entre $20 \%$ a $47 \%$ (3). Debido a su baja incidencia y las dificultades en la terapéutica, el objetivo de esta revisión se basa en exponer los datos más recientes sobre la fisiopatología, etiología, clasificación, manifestaciones clínicas, diagnóstico y tratamiento de esta entidad para el manejo adecuado y oportuno.

\section{Métodos}

La búsqueda para la revisión sistemática de este tema se hizo en la base de datos como PubMed, UpToDate, The Cochrane y Scielo. Los criterios de inclusión fueron publicaciones en publicaciones recientes, con las palabras clave "fascitis necrotizante", "infección de tejidos blandos" y "fascia", en el idioma español e ingles. Se revisaron 14 artículos que cumplen con los puntos esenciales sobre el tema.

\section{Etiología}

La fascitis necrotizante se puede dividir en dos categorías según los microorganismos causales: polimicrobiana (Tipo I) y monomicrobiana (Tipo II). La tipo I, siendo la más frecuente (4), es una infección causada por especies de anaerobios (comúnmente Bacteroides, Clostridium o Peptostreptococcus) en combinación con enterobacterias (como E. coli, Enterobacter, Klebsiella y Proteus) y uno o más estreptococos facultativos. Los hongos y aerobios obligatorios, como Pseudomonas aeruginosa, son infrecuentes de encontrar en estos casos. En la tipo II, la causa más común es por estreptococo del grupo A u otro estreptococo betahemolítico. También, puede ser causado por Staphylococcus aureus. Organismos causales menos frecuentes incluyen Vibrio vulnificus y Aeromonas hydrophila (5).

Dentro de las complicaciones más frecuentes, la gangrena de Fournier, que se presenta en el periné, es causada por organismos facultativos (E.coli, Klebsiella y enterococos) junto con anaerobios (Bacteroides, Fusobacterium, Clostridium, anaerobios o estreptococos microaerofílicos). En el caso de la infección necrótica en cabeza y cuello, la causa más frecuente son anaerobios de la mucosa oral (como Fusobacterias, estreptococos anaerobios, Bacteroides, y espiroquetas). El estreptococo beta-hemolítico es que agente causal más frecuente en neonatos (5).

\section{Factores de riesgo}

Este padecimiento se puede diagnosticar tanto en individuos sanos como en portadores de enfermedades crónicas, en cualquier grupo de edad. Sin embargo, es más frecuente entre 40 y 70 años de edad, con cierto predominio en hombres $(5,6)$. Otros factores de riesgo incluyen, trauma (mayor o menor), heridas en piel, cirugía reciente, diabéticos, alcohólicos, inmunosupresos, obesos, pacientesoncológicos, malnutrición, con enfermedades vasculares periféricas e insuficiencia renal y hepática (7). En mujeres, particularmente se encuentra en embarazadas, con un parto reciente, aborto o procedimiento ginecológico reciente (8). 


\section{PATOGENIA}

El proceso de infección inicia en la fascia superficial, donde se genera un crecimiento y proliferación bacteriana, de toxinas y enzimas que generan una disrupción de la misma generando progresión a profundidad de los tejidos.

Esta proliferación también produce una liberación de citoquinas que generan necrosis de la fascia superficial y trombosis de la red vascular adyacente $(9,10)$. Al existir este compromiso necrótico asociado a infección se afectan los planos más profundos y la dermis, persentandose los signos clínicos locales característicos de la enfermedad: necrosis cutánea, ulceración y presencia de lesiones ampollosas (11).

\section{MANIFESTACIONES CLÍNICAS}

La FN se caracteriza por presentar inicialmente un cuadro inespecífico de dolor fuera de proporción al edema y eritema presente. Sin embargo esta presentación puede verse afectada en paciente que hayan recibido analgesia, padecen de neuropatía diabética, o tengan alteración del estado de consciencia. Conforme el cuadro evoluciona se desarrollan ampollas y bullas quienes son intermediarias entre la fase temprana y la fase tardía (Tabla 1).

Por lo tanto todo paciente que se presente con dolor de inicio súbito en una extremidad, con o sin un portal de entrada bacteriano aparente con o sin presencia de fiebre se debe realizar el diagnóstico diferencial de FN (12).

\begin{tabular}{|c|c|c|}
\hline $\begin{array}{l}\text { Estadío } 1 \\
\text { (temprano) }\end{array}$ & $\begin{array}{c}\text { Estadío } 2 \\
\text { (intermedio) }\end{array}$ & $\begin{array}{l}\text { Estadío } 3 \\
\text { (tardío) }\end{array}$ \\
\hline $\begin{array}{c}\text { Calor a la } \\
\text { palpación } \\
\text { Eritema } \\
\text { Dolor a la } \\
\text { palpación (más } \\
\text { allá de las } \\
\text { áreas } \\
\text { apatentes en } \\
\text { piel) } \\
\text { Edema }\end{array}$ & $\begin{array}{c}\text { Ampollas o } \\
\text { bullas (líquido } \\
\text { seroso) } \\
\text { Fluctuación de } \\
\text { piel } \\
\text { Induración de } \\
\text { piel }\end{array}$ & $\begin{array}{c}\text { Bullas } \\
\text { hemorrágias } \\
\text { Anestesia al } \\
\text { tacto } \\
\text { Crépitos } \\
\text { Necrosis de la } \\
\text { piel con } \\
\text { descoloración } \\
\text { y progesión a } \\
\text { gangrena }\end{array}$ \\
\hline
\end{tabular}

Fuente: Adaptado de Tabla 3 "Evolution of physical signs of necrotizing fasciitis from early to late disease". Tomado del artículo Early diagnosis of necrotizing fasciitis" de John Wiley \& Sons Ltd.

\section{DIAGNÓSTICO}

Importancia del diagnóstico temprano: en la FN el diagnóstico temprano es primordial para disminuir la morbimortalidad que genera un abordaje tardío. Este fallo en el diagnóstico temprano se debe diversos factores clínicos, como lo son: la ausencia de fiebre, esto se debe por el uso de antiinflamatorios no esteroideos (AINES) para el manejo del dolor que enmascaran este signo; cuando el agente causal es C.sordelli con frecuencia la fiebre se encuentra ausente; otro factor, es la ausencia de manifestaciones cutáneas ya que el sitio de entrada bacteriano puede no ser evidente y sus manifestaciones se presentan de forma tardía. Por otra parte en los estudios por imágenes, las radiografías suelen mostrar únicamente edema sin evidencia de gas en los tejidos blandos, lo

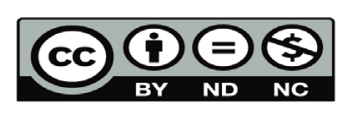


que tienen a confundir en el diagnóstico (13).

Estos paciente usualmente se presentan con la triada de dolor, edema y eritema, la cual es inespecífica y erróneamente se puede diagnosticar como celulitis o absceso. Una característica clínica determinante de la fascitis necrotizante es el dolor fuera de propoción al eritema y/o edema que se encuentra presente (14). Las cuatro características diferenciantes de la FN de una infección simple de tejidos blandos son: el dolor que se extiende más allá del área por la diseminación de toxinas; en la FN los márgenes comprometidos no son definidos; la linfangitis rara vez se encuentra presente ya que la infección se encuentra en la fascia produnfa y no en la piel; y por últimoen la FN la enfermedad progesa rápidamente a pesar del uso de antibióticos (12).

Estudios por imágenes: Las técnicas por imagen pueden ser útiles para orientar el diagnóstico en una infección necrotizante, sin embargo, este no debe retrasar la intervención quirúrgica cuando se la clínica sugiera el diagnóstico de $\mathrm{FN}$, cuya tasa de falsos negativos es elevada.

- Tomografía axial computarizada (TAC): es el estudio de imagen por elección, dada su disponibilidad y rápida realización del estudio; los hallazgos que se puede observar son: engrosamiento y edema de la fascia profunda con presencia de gas en los planos superficiales $(13,14)$.

- Resonancia magnética: tiene mayor sensibilidad para la valoración de tejidos blando en comparación con el TAC, pero la disponibilidad de este estudio es limitada y la duración de la realización del estudio prolongada por lo que no está indicada $(13,14)$.
Biopsia y tinción de Gram: La tinción de Gram del material obtenido quirúrgicamente es crucial para determinar la causa de la infección y guiar el tratamiento empírico. Por otra parte se realiza una inspección quirúrgica abierta con toma de biopsia (15). es de vital importancia. Las características histológicas por Esteptococo B-hemolítico del Grupo A son: destrucción del tejido muscular, pocas células inflamatorias y un gran número de cocos gram positivos en el sitio (16).

Por otra parte, para un abordaje más sistemático de la FN el algoritmo (Esquema 1.1), consiste de los siguientes apartados: la sospecha clínica ante la presencia de síntomas (edema, eritema y dolor), signos sistémicos que presente el paciente (taquicardia > $120 \mathrm{lpm}$, hipotensión, niveles de CK elevados, escala LRINEC (Laboratory Risk Indicator for Necrotizing Fasciitis) $>6$ ); si el paciente presenta alguno de estos se debe realizar estudios de imagen para detectar si hay presencia de gas o no, esto nos va a guiar en los posibles patógenos que están causando la enfermedad. Independientemente de si hay o no presencia de gas, se debe realizar una inspección y desbridamiento, además de tomar un cultivo del tejido afectado.

Si se evidencia la presencia de gas en los estudios por imagen se debe sospechar que en el cultivo tomado anteriormente van a evidenciar bacilos Gram positivos o bacterias aerobias junto a anaerobias. Si no existe la presencia de gas, se debe sospechar la presencia de Cocos Gram positivos o Bacilos Gram negativos. Si el inicialmente no presenta signos sistémicos se debe realizar diagnóstico diferencial con otras patologías (celulitis, erisipela, absceso cutáneo). (12). 


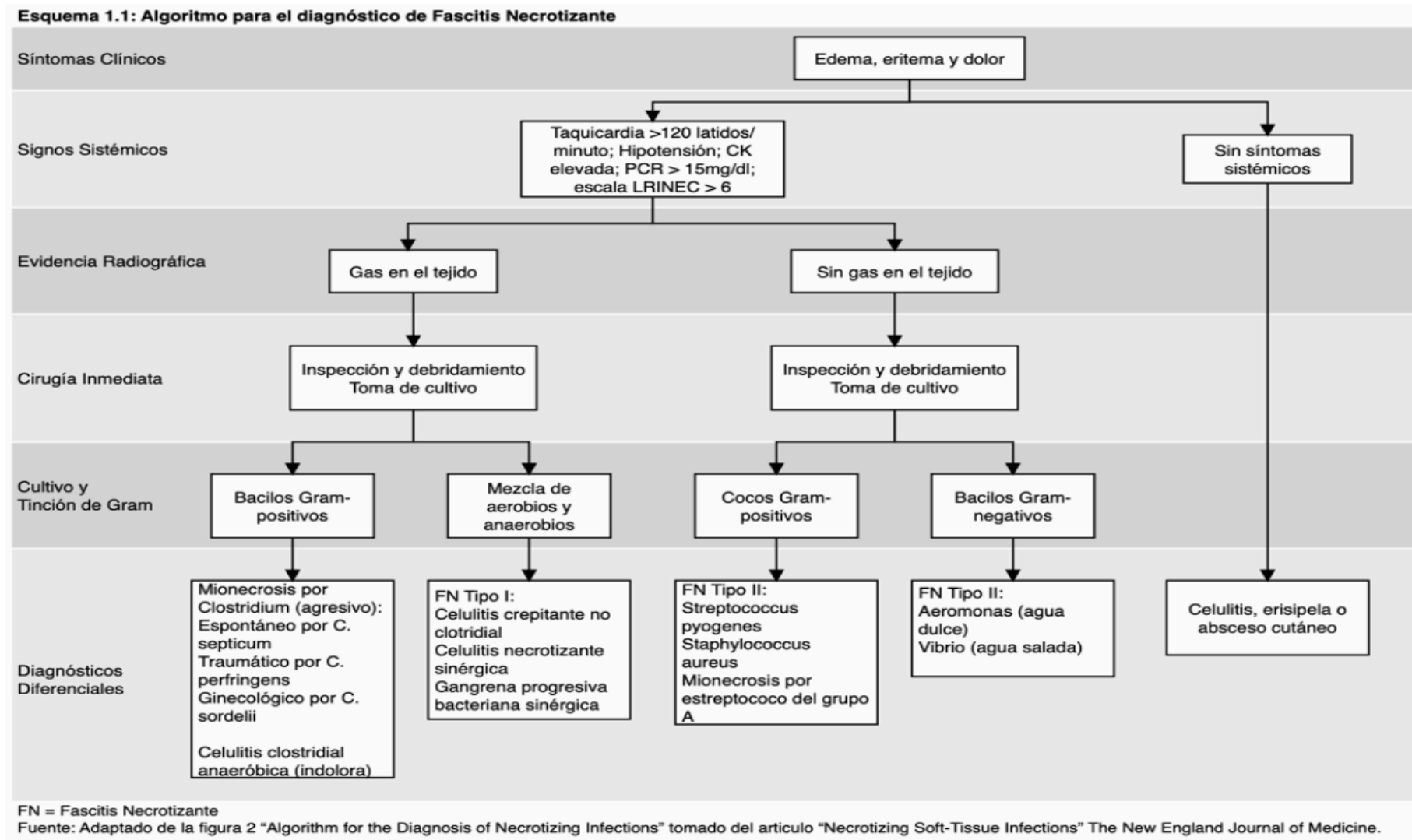

\section{TRATAMIENTO}

El manejo en ocasiones es complicado debido a su rápida progresión. El tratamiento se basa en tres intervenciones principales: el soporte hemodinámico, la desbridación quirúrgica y uso de antimicrobiamos. Siempre que se sospeche un diagnóstico de fascitis necrotizante, la exploración por el cirujano está indicada lo antes posible para realizar el desbridamiento con el equipo de cirugía en la medida que se considere necesario según la apariencia de los tejidos, o para confirmar el diagnóstico, si sigue en cuestión. Además, es de gran ayuda los estudios de imagen tempranamente (8).

-Soporte Hemodinámico: Si el paciente presenta con inestabilidad hemodinámica inicialmente maneja de manera agresiva con fluidos, y en algunos casos hasta vasopresores (estado de choque o disfunción orgánica) $(7,8$, 16).

- Tratamiento quirúrgico: El pilar para el pronóstico es un manejo quirúrgico inicial con desbridación para lograr una reducción en la morbimortalidad del paciente. La gravedad de la infección causa con frecuencia un deterioro a las pocas horas de la presentación inicial, y tiene el potencial de causar necrosis extensa de tejidos blandos y estructuras adyacentes, demostrando la importancia de un desbridamiento agresivo y cuidados críticos de apoyo; seguidos de un largo procedo se reconstrucción y rehabilitación para recuperar la forma y función del área afectada. $(8,16)$. Se debe desbiridar el tejido necrótico hasta alcanzar un tejido sano y viable, que se identifica con la aparición de sangre rutilante. Es usual que ocurra trombosis en la red capilar causando necrosis cutánea y de tejidos con 
mayor profundidad (8). Se deben explorar el margen y la profundidad de la herida, inclusive fascia profunda y músculo para garantizar que menor grado de extensión. Es recomendable realizar evaluaciones quirúrgicas al menos 24 horas después del procedimiento inicial y repetir debridaciones hasta conseguir eliminar el tejido necrótico e infectado (17).

- Tratamiento antimicrobiano: Es necesario empezar con una cobertura de antibióticos de amplio espectro que incluya actividad contra bacterias gram-positivas, gram-negativas y anaerobios $(8,14)$. Previo al inicio de antibioticoterapia se recomienda tomar cultivos para valorar sensibilidad antibiótica. Mientras se obtienen lo resultado del cultivo, se debe comenzar con un régimen generalizado que consiste en carbapenémicos (imipenem, meropenem, ertapenem) o piperacilinatazobactam más un agente contra $S$. aureus meticilin-resistente (como vancomicina o daptomicina) más clindamicina (por su acción antitoxina y otros efectos contra cepas que producen toxinas estreptococos y estafilococos) (15).

Al identificar el o los organismos causales, se puede adaptar el tratamiento.

- Fascitis necrotizante polimicrobiana (Tipo I): combinación de ampicilina o ampicilinasulbactam más clindamicina o metronidazol (7).

- Fascitis necrotizante monomicrobiana (Tipo II): se recomienda la administración de penicilina $\mathrm{G}$ más clindamicina (7).

Un aspecto importante a considerar es el mecanismo de la lesión y la exposición que el paciente pudo tener que sugiera una inoculación por organismos específicos, por ejemplo, Aeromonas en el caso de agua dulce o Vibrio vulnificus en agua salada, para así poder optimizar la cobertura antibiótica.

\section{- OTROS TRATAMIENTOS:}

- Amputación: Se debe considerar cuando es poco probable que los desbridamientos repetidos obtengan el control de la fuente infecciosa, cuando es probable que los resultados funcionales sean obviamente mejores en comparación con una reconstrucción, o cuando no existan opciones reconstructivas. Además, se ha visto más frecuentemente indicado para pacientes críticos con diabetes mellitus, enfermedad arterial periférica subyacente 0 infección por estreptococos del grupo $A(8,17)$.

- Terapia con presión negativa (VAC): Con esta terapia, se mantiene un ambiente húmedo y cerrado de la herida, ayuda con el exceso de exudado y a preparar un lecho de la herida para injertos de piel o colgajos de tejido, debido a que estimula la formación de tejido de granulación. Al usarlo, posterior a la desbridación inicial, las cirugías siguientes se pueden llevar a cabo con mayores intervalos de tiempo entre sí, y ayuda para la antisepsia continua y la preparación del lecho de la herida para el manejo posterior (8, 18).

- Oxígeno Hiperbárico: La evidencia para su uso en la fascitis necrotizante es controversial debido a que puede haber un beneficio en términos de recuperación de extremidades; sin embargo, el traslado del paciente a un centro hospitalario con esta terapia puede comprometer el pronóstico del paciente. Su efecto más estudiado es que que podría inhibir la producción de exotoxinas y mejorar la capacidad fagocítica 
de los neutrófilos (7).

- Manejo del dolor: Una atención inadecuada al dolor perjudica significativamente la rehabilitación física y psicosocial y aumenta el riesgo de alodinia o hiperalgesia del área afectada. El paciente experimenta tres tipos de dolor:

-Dolor de fondo: es causado por la patología de fondo y se maneja con agentes narcóticos de larga duración, como morfina, y acetaminofén o antiinflamatorios no esteroideos (19).

-Dolor irruptivo: el que se exacerba por terapia, emociones o un simple movimiento del área afectada y es manejado con opioides de duración corta (8).

-Dolor por procedimientos: es el resultado de las intervenciones quirúrgicas o cambios de apósitos en la herida, se trata con ansiolíticos de acción corta como midazolam más opiáceas de acción corta intravenosos como fentanilo o morfina (19).

\section{CONCLUSIÓN}

La fascitis necrotizante es una enfermedad infecciosas de elevada morbimortalidad si no se diagnostica a tiempo. La reducción del riesgo se centra en un abordaje con intervención quirúrgica urgente, agresiva, y antibioticoterapia sistémica. El manejo del paciente debe ser integral, no solo por parte del médico, si no también los aspectos nutricionales, el soporte psicológico, familiar, y la eventual rehabilitación de la lesión. Cabe resaltar, que el pronóstico del paciente y su tratamiento adecuado dependerá de la ayuda de las imágenes médicas tempranas y una buena labor en conjunto con el equipo quirúrgico de manera precoz.

\section{BIBLIOGRAFÍA}

1. T. Goh, L. G. Goh, C. H. Ang, C.H.Wong. Early diagnosis of necrotizing fasciitis. [Internet]. BJ Surgery Society. 2014 Jan;101(1):e119-25. doi:10.1002/bjs.9371 [Consultado 05 Junio, 2020]; Disponible en: https://pubmed.ncbi.nlm.nih.gov/24338771

2. F Carbonetti, V Carusi, M Guidi,V David. Necrotizing Fasciitis: A Comprehensive Review. [Internet]. Clin Ter 2015;166(2):e132-9. doi: 10.7417/CT.2015.1836. [Consultado 05 Junio, 2020]. Disponible en: https://pubmed.ncbi.nlm.nih.gov/25945447

3. M Leiblein, I Marzi, A L Sander, J H Barker, F Ebert, J Frank. Necrotizing Fasciitis: Treatment Concepts and Clinical Results. Br J Surg 2014 Jan;101(1):e119-25. doi: 10.1002/bjs.9371. Disponible en: https://pubmed.ncbi.nlm.nih.gov/28484782

4. Krieg A, Röhrborn A, Schulte am Esch J, Schubert D, Poll LW, Ohmann C, Braunstein S, Knoefel WT. Necrotizing Fasciitis: Micorbiological characteristics and predictors of postoperative outcome [Internet]. Eur J Med Res. 2009; 14(1): 30-36. [PMC free article] [PubMed] [Consultado 05 Junio, 2020]; Disponible en https://www.ncbi.nlm.nih.gov/pmc/articles/PMC3352202/

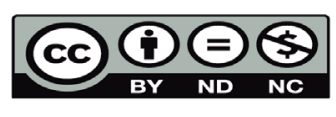


5. Taro Shimizu, Yasuharu Tokuda. Necrotizing Fasciitis. Intern Med 2010;49(12):1051-7. doi: 10.2169/internalmedicine.49.2964. [Internet]. Eur J Med Res. 2009; 14(1): 30-36. [PMC free article] [PubMed] [Consultado 05 Junio, 2020]; Disponible en: https://pubmed.ncbi.nlm.nih. gov/20558917

6. Stevens DL , Baddour LM . Necrotizing soft tissue infections. Up to Date [Internet]. Actualizado el 28 de Febrero 2020, consultado el 04 Junio, 2020.Burnham JP, Kollef MH. Treatment of severe skin and soft tissue infections: A review. Curr Opin Infect Dis. 2018;31:113-9. [PMC free article] [PubMed] [Google Scholar] [Consultado 04 Junio, 2020]; Disponible en: https://www. ncbi.nlm.nih.gov/pmc/articles/PMC6200137/

7. Hernández González EH, Mosquera Betancourt CG, de la Rosa Salazar V. Fascitis necrotizante [Internet] Revista Archivo Médico de Camagüey en 2015 [Consultado 04 Junio, 2020]; Disponible en: http://scielo.sld.cu/scielo.php?script=sci_arttext\&pid=S1025-02552015000600012\&lan$g=e n$

8. Rogers AD, Shahrokhi S. Surgical management of necrotizing soft tissue infections. Up to Date [Internet]. Actualizado el 12 de Agosto 2019, consultado el 04 Junio, 2020.

9. Rukshini Puvanendran, Jason Chan Meng Huey, Shanker Pasupathy. Necrotizing Fasciitis. Can Fam Physician 2009 Oct;55(10):981-7.[Consultado 04 Junio, 2020]; Disponible en: https:// pubmed.ncbi.nlm.nih.gov/19826154

10. Chin-Ho Wong, Yi-Shi Wang. The Diagnosis of Necrotizing Fasciitis. [Internet] Curr Opin Infect Dis. 2005 Apr;18(2):101-6. doi: 10.1097/01.qco.0000160896.74492.ea. [Consultado 04 Junio, 2020]; Disponible en: https://pubmed.ncbi.nlm.nih.gov/15735411/

11. F G Barker, B J Leppard, D V Seal. Streptococcal Necrotising Fasciitis: Comparison Between Histological and Clinical Features. [Internet] J Clin Pathol 1987 Mar;40(3):335-41. doi: 10.1136/jcp.40.3.335. Consultado [05 Junio, 2020]. Disponible en: https://pubmed.ncbi.nlm.nih. gov/3558868/

12. Steves DL, Bryant AE. Necrotizing Soft-Tissue Infections. The New England Journal of Medicine [Internet] N Engl J Med 2017; 377:2253-2265. Consultado el 05 Junio 2020.

13. Frazee B, Fee C, Lynn J, Wang R, Bostrom A, Hargis C, Moore P. Community-acquired necrotising soft tissue infections: a review of 122 cases presenting to a single emergency department over 12 years. J Emerg Med 2008; 34: 139 - 146.

14. Hsiao $\mathrm{C}$, Weng $\mathrm{H}$, Yuan $\mathrm{Y}$, Chen $\mathrm{C}$, Chen I. Predictors of mortality in patients with necrotizing fasciitis. Am J Emerg Med 2008; 26: 170 - 175. 
15. Stamenkovic I, Lew PD. Early recognition of potentially fatal necrotizing fasciitis the use of frozen-section biopsy. N Engl J Med 1984;310:1689-1693

16. Majeski J, Majeski E. Necrotizing fasciitis: improved survival with early recognition by tissue biopsy and aggressive surgical treatment. South Med J 1997;90:1065-1068

17. P. P. Caballero, S. Pérez, M. Patiño, S. Castañeda, J.García. Actualización en fascitis necrotizante. [Internet]. Elsevier, Seminarios de la Fundación Española de Reumatología 41-48 (Abril - Junio 2012). [Consultado 05 Junio, 2020]. Disponible en:https://www.elsevier.es/es-revista-seminarios-fundacion-espanola-reumatologia-274-articulo-actualizacion-fascitis-necrotizante-S1577356611001205\#bib0210

18. Burnham JP, Kollef MH. Treatment of severe skin and soft tissue infections: a review [Internet]. Curr Opin Infect Dis. 2018 April ; 31(2): 113-119. doi:10.1097/QCO.0000000000000431. [Consultado 04 Junio 2020]. Disponible en https://www.ncbi.nlm.nih.gov/pmc/articles/PMC6200137/

19. Stevens DL , Baddour LM . Necrotizing soft tissue infections. Up to Date [Internet]. Actualizado el 28 de Febrero 2020, consultado el 04 Junio, 2020. 